\title{
A small pancreatic hamartoma with an obstruction of the main pancreatic duct and avid FDG uptake mimicking a malignant pancreatic tumor: a systematic case review
}

\author{
Hiroaki Nagano ${ }^{1 *}$, Masayuki Nakajo ${ }^{1}$, Yoshihiko Fukukura ${ }^{5}$, Yoriko Kajiya ${ }^{1}$, Atsushi Tani ${ }^{1}$, Sadao Tanaka ${ }^{2}$, \\ Mari Toyota ${ }^{3}$, Toru Niihara ${ }^{3}$, Masaki Kitazono ${ }^{4}$, Toyokuni Suenaga ${ }^{4}$ and Takashi Yoshiura ${ }^{5}$
}

\begin{abstract}
Background: Pancreatic hamartomas are extremely rare and may be misdiagnosed as malignant tumors. We report herein a case of a small, solid-type pancreatic hamartoma.

Case presentation: A 72-year-old female was incidentally detected pancreatic lesion by ultrasonography. Computed tomography and magnetic resonance imaging revealed a 2.0-cm solid lesion. The main pancreatic duct (MPD) was obstructed by the lesion in the head of the pancreas, and the upstream MPD was dilated. ${ }^{18} \mathrm{~F}$-fluorodeoxyglucose (FDG) accumulated avidly in the lesion and increased in FDG intensity from the early to the delayed images. The histopathological studies confirmed the diagnosis of pancreatic hamartoma. Immunohistochemically, the cell membrane of the accessory glands and ducts showed homogeneous expression of glucose transporter type I and hexokinase II.
\end{abstract}

Conclusion: Pancreatic hamartomas causing dilatation of the MPD are extremely rare, and this appears to be the first case of a hamartoma to take up FDG avidly. It was a rare occurrence and should be noted that pancreatic hamartomas can cause an obstruction of the MPD and show avid FDG uptake, thereby mimicking malignant pancreatic tumors.

Keywords: Hamartoma, Pancreas, CT, MRI, FDG, PET/CT

\section{Background}

Hamartomas are benign, tumor-like nodules composed of an overgrowth of mature cells and tissues that normally occur in the affected tissue, but often with one predominant element. Pancreatic hamartomas are composed of three disarranged cellular components in varying proportions: acinar, islet, and ductal cells [1]. These hamartomas are extremely rare and may be misdiagnosed as malignant tumors. Herein, we report a case of pancreatic hamartoma resembling a malignant pancreatic tumor.

\footnotetext{
* Correspondence: hi-naga@m3.kufm.kagoshima-u.ac.jp

'Departments of Radiology, Nanpuh Hospital, 14-3 Nagata, Kagoshima 892-8512, Japan

Full list of author information is available at the end of the article
}

\section{Case presentation}

A 72-year-old asymptomatic woman was referred to our hospital for examination of a pancreatic lesion detected on abdominal ultrasonography during a health screening. Her past medical history and physical examination were unremarkable. On laboratory testing, the serum levels of amylase, bilirubin, carcinoembryonic antigen, and carbohydrate antigen 19-9 were within normal limits.

Abdominal ultrasonography revealed a heterogeneous, hypoechoic mass in the pancreatic head. Dynamic computed tomography $(\mathrm{CT})$ demonstrated a non-deforming mass in the pancreatic head measuring $2.0 \mathrm{~cm}$ in maximum diameter and a dilated upstream of the main pancreatic duct (MPD) (Fig. 1a and b). The lesion 


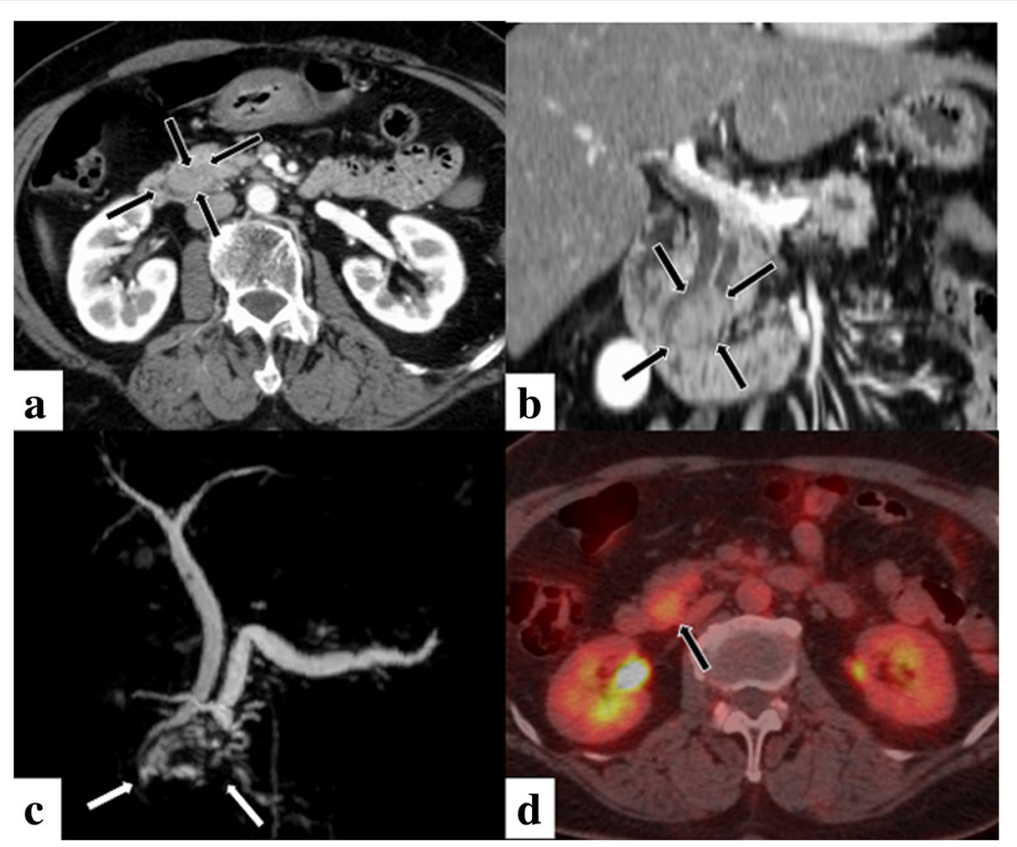

Fig. 1 Contrast-enhanced CT axial (a) and coronal (b) images demonstrates a $2.0 \mathrm{~cm}$, non-deforming mildly hypoattenuating mass in the pancreatic head (arrows) associated with obstruction of the pancreatic duct. MRCP (c): Confirms the dilatation of the pancreatic duct (8 mm in diameter) in the body of the pancreas. Note the associated dilatation of the Santorini duct (small arrows). FDG-PET/CT (d): The pancreatic lesion (arrow) shows avid ${ }^{18}$ F-fluorodeoxyglucose (FDG) uptake on the early image. The lesion increases in visual intensity on the delayed image on FDG-PET/CT

showed isoattenuation on unenhanced CT, mild hypoattenuation during the pancreatic parenchymal phase, and isoattenuation during the portal venous and delayed phases relative to the surrounding pancreatic parenchyma. On magnetic resonance imaging (MRI), the lesion showed heterogeneous low-signal intensity on T1weighted imaging and mild high-signal intensity on T2-weighted imaging compared to the surrounding pancreatic parenchyma. On diffusion-weighted imaging, the lesion showed mild high-signal intensity with an apparent diffusion coefficient value of $1.4 \times 10^{-3} \mathrm{~mm}^{2} / \mathrm{s}$. On MR cholangiopancreatography (MRCP) (Fig. 1c), the lesion obstructed the MPD in the head of the pancreas, and the upstream pancreatic duct was smoothly dilated (8 $\mathrm{mm}$ in diameter). Endoscopic retrograde cholangiopancreatography (ERCP) showed a stricture of the MPD with a dilated upstream. Endoscopic ultrasound (EUS) showed a $24 \mathrm{~mm}$ mural hyperechoic mass in the MPD. On positron emission tomography/CT (PET/CT), the pancreatic lesion showed avid ${ }^{18} \mathrm{~F}$-fluorodeoxyglucose (FDG) uptake with a maximum standardized uptake value (SUVmax) of 3.6 at the early imaging ( $1 \mathrm{~h}$ after intravenous FDG injection) (Fig. 1d), which increased to 5.0 at the delayed imaging ( $2 \mathrm{~h}$ after intravenous FDG injection).

The patient then underwent a subtotal pyloricpreserving pancreaticoduodenectomy. Macroscopically, a well-demarcated, homogeneous, white-to-yellow-colored solid lesion measuring $2.0 \mathrm{~cm}$ was noted in the pancreas head. Microscopically, the solid lesion was composed of accessory glands and ducts, lined by cuboidal to flattened epithelium without atypia (Fig. 2a). The accessory glands and ducts were embedded in a fibrotic stroma, and the islets of Langerhans were not evident within the lesion. Lymphocyte and follicle formation infiltrated diffusely around the MPD. Immunohistochemically, the ductal cells were positive for epithelial markers, but negative for synaptophysin and chromogranin A. The cell membrane of the accessory glands and ducts showed homogeneous expression of glucose transporter type I (GLUT-1) (Fig. 2b) and hexokinase II (HK-II). The expressions were especially strong along the basilar membrane of the accessory glands. The pathological diagnosis was solid-type pancreatic hamartoma. After surgical resection, the patient received follow-up CT examinations every three to 6 months and there has been no recurrence for 36 months.

\section{Discussion}

Pancreatic hamartomas are extremely rare, and their radiologic features have not been fully evaluated. To the best of our knowledge, there have been only 30 previous reports of pancreatic hamartomas in the literature, and there are very few reports regarding their imaging appearance [1-20]. Age at presentation ranged from 34 weeks to 78 years (mean, 48.6 years). Both sexes were 


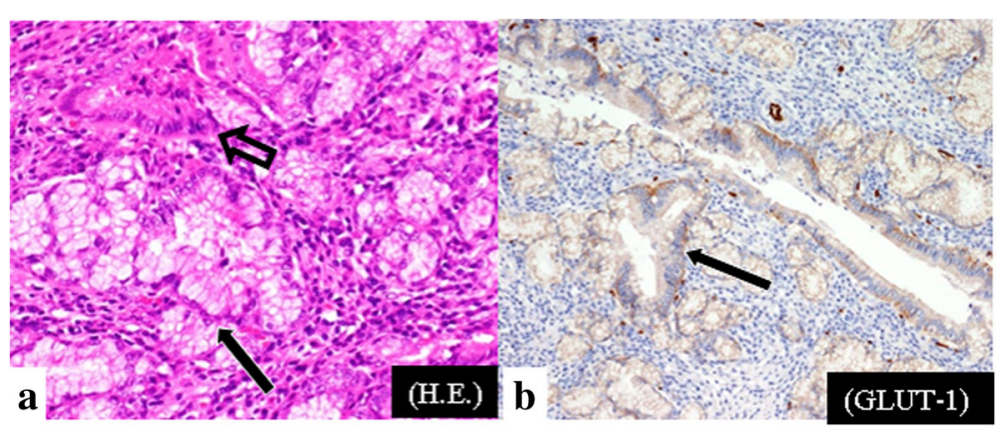

Fig. 2 Macroscopic and immunohistochemical findings. a: The lesion is composed of accessory glands (closed arrows) and ducts lined by cuboidal to flattened epithelium, without atypia (open arrows). b: On immunohistochemistry, the cell membrane of the accessory glands and ducts show homogeneous expression of glucose transporter type I. The expressions are especially strong along the basilar membrane of the accessory glands (closed arrows)

equally affected, with a male-to-female ratio of 1:0.82. Most patients were either asymptomatic or they exhibited symptoms related to local mass effect, including abdominal pain, weight loss, and a palpable mass; and only one patient had jaundice, caused by obstruction of the common bile duct [17]. Pancreatic hamartomas were reported to arise from all parts of the pancreas, although the pancreatic head was the most common site $(64.5 \%$, 20/31). Their size ranged from $1.0-14.0 \mathrm{~cm}$ (mean, $4.5 \mathrm{~cm})$.

Morphologically, there are two types of pancreatic hamartoma: solid, and solid and cystic [2, 3]. Nine of the previously reported cases of pancreatic hamartoma seemed to fit the criteria of solid type; however, only three reports included the radiological appearance $[1,16,17]$. One showed that the MPD was compressed by the hamartoma located in the pancreatic body, and the upstream was dilated [16]. The remaining two cases had no stricture or dilatation of the MPD [1, 17]. On enhanced CT, one case showed a hyperattenuating hamartoma during the portal venous phase [17], and the other two cases demonstrated delayed enhancement on contrast-enhanced CT or MRI $[1,16]$. In the present case, a pancreatic hamartoma measuring $2.0 \mathrm{~cm}$ in diameter was located in the head of the pancreas and caused an obstruction of the MPD, with a dilated upstream on CT, MRCP, and ERCP. Contrast-enhanced CT demonstrated an ill-defined lesion with mild hypoattenuation during the pancreatic parenchymal phase and isoattenuation during the portal venous and delayed phases.

Pancreatic ductal adenocarcinomas may present as a non-deforming iso- or hypoattenuation mass during the pancreatic parenchymal to portal venous phase on enhanced CT [21], and have an obstruction of the MPD with upstream ductal dilatation on CT or MRI [21, 22]. Pancreatic neuroendocrine tumors usually show hypervascular patterns during the arterial to the portal venous phase.
However, high-grade pancreatic neuroendocrine tumors tend to show hypovascular patterns and an interruption of the MPD with upstream ductal dilatation [23-25]. Therefore, in the present case, it was difficult to differentiate pancreatic hamartoma from ductal adenocarcinoma, neuroendocrine tumor, or pancreatic metastases.

On FDG-PET, no avid FDG uptake was noted in the three previously reported cases of pancreatic hamartoma $[9,14,15]$. Ours appears to be the first case of a pancreatic hamartoma to show avid FDG uptake, which may be related to the GLUT-1 and HK-II expression of the accessory glands and ducts. Yoshioka et al. [26] reported that an FDG SUVmax of 2.5 would be justified as a cutoff value to differentiate between malignant and benign pancreatic tumors. Kawada et al. [27] reported that the FDG SUVmax increased from the early to the delayed images in $89 \%(39 / 44)$ of the small malignant pancreatic tumors $(<25 \mathrm{~mm})$. In the present case, the FDG SUVmax was 3.6 at the early phase and increased to 5.0 at the delayed phase, in concordance with malignant pancreatic tumors. In our case, therefore, it was difficult to differentiate from malignant tumors based on US, CT, MRI, and FDG-PET/CT findings. Pancreatic hamartoma is benign entity, and does not require surgical treatment. EUS-Fine-Needle-Aspiration might have been effective for definitive diagnosis if we had performed it.

\section{Conclusion}

Pancreatic hamartomas causing dilatation of the MPD were extremely rare, and showed no avid FDG uptake in the previously reported cases. We described a rare case of a small, solid-type pancreatic hamartoma that showed an obstruction of the main pancreatic duct and avid FDG uptake. Radiologists should be aware that pancreatic hamartomas may be associated with these findings.

\section{Abbreviations}

CT: Computed tomography; ERCP: Endoscopic retrograde

cholangiopancreatography; EUS: Endoscopic ultrasound; FDG: ${ }^{18} \mathrm{~F}-$ 
fluorodeoxyglucose; GLUT-1: Glucose transporter type I; HE: Hematoxylineosin staining; HK-II: Hexokinase II; MPD: Main pancreatic duct; MRCP: MR cholangiopancreatography; MRI: Magnetic resonance imaging; PET/ CT: Positron emission tomography/CT; SUVmax: Maximum standardized uptake value

\section{Acknowledgements \\ Not applicable.}

\section{Funding}

The authors have no funding to report.

\section{Availability of data and materials}

The datasets supporting the conclusions of this article are included within the article.

\section{Authors' contributions}

All authors participated in clinical examinations, diagnosis, surgical operation and follow up for of this patient. MN, YF, YK, AT and TY took part in the design of this study and helped to draft the manuscript. All authors read and approved the final manuscript

\section{Ethics approval and consent to participate}

This case report was granted an exemption from requiring ethics approval by the Institutional Review Board of Nanpuh Hospital.

\section{Consent for publication}

Written informed consent was obtained from the patient for publication of this case report and any accompanying images. A copy of the written consent is available for review by the Editor of this journal.

\section{Competing interests}

The authors declare that they have no competing interests.

\section{Publisher's Note}

Springer Nature remains neutral with regard to jurisdictional claims in published maps and institutional affiliations.

\section{Author details}

'Departments of Radiology, Nanpuh Hospital, 14-3 Nagata, Kagoshima 892-8512, Japan. ${ }^{2}$ Departments of Pathology, Nanpuh Hospital, 14-3 Nagata, Kagoshima 892-8512, Japan. ${ }^{3}$ Departments of Gastroenterology, Nanpuh Hospital, 14-3 Nagata, Kagoshima 892-8512, Japan. ${ }^{4}$ Departments of Surgery, Nanpuh Hospital, 14-3 Nagata, Kagoshima 892-8512, Japan. ${ }^{5}$ Department of Radiology, Kagoshima University Graduate School of Medical and Dental Sciences, 8-35-1 Sakuragaoka, Kagoshima-shi, Kagoshima 890-8544, Japan.

Received: 5 July 2016 Accepted: 24 November 2017

Published online: 06 December 2017

\section{References}

1. Nagata S, Yamaguchi K, Inoue T, Yamaguchi H, Ito T, Gibo J, et al. Solid pancreatic hamartoma. Pathol Int. 2007;57:276-80.

2. Pauser U, Kosmahl M, Kruslin B, Kilmstra DS, Klöppel G. Pancreatic solid and cystic hamartoma in adults: characterization of a new tumorous lesion. Am J Surg Pathol. 2005;29:797-800.

3. Pauser U, da Silva MT, Placke J, Kilmstra DS, Klöppel G. Cellular hamartoma resembling gastrointestinal stromal tumor: a solid tumor of the pancreas expressing c-kit (CD117). Med Pathol. 2005;18:1211-6.

4. Anthony PP, Faber RG, Russell RC. Pseudotumours of the pancreas. Br Med J. 1977;1:814.

5. Burt TB, Condon VR, Matlak ME. Fetal pancreatic hamartoma. Pediatr Radiol. 1983:13:287-9.

6. Flaherty MJ, Benjamin DR. Multicystic pancreatic hamartoma: a distinctive lesion with immunohistochemical and ultrastructural study. Hum Pathol. 1992;23:1309-12.

7. Izbicki JR, Knoefel WT, Müller-Höcker J, Mandelkow HK. Pancreatic hamartoma: a benign tumor of the pancreas. Am J Gastroenterol. 1994;89: 1261-2.

8. Wu SS, Vargas HI, French SW. Pancreatic hamartoma with Langerhans cell histiocytosis in a draining lymph node. Histopathology. 1998;33:485-7.
9. McFaul CD, Vitone LJ, Campbell F, Azadeh B, Hughes ML, Garvey CJ, et al. Pancreatic hamartoma. Pancreatology. 2004:4:533-8.

10. Thrall M, Jessurun J, Edwaard B, Stelow N, Adsay V, Selwyn M, et al. Multicystic adenomatoid hamartoma of the pancreas: a hitherto undescribed pancreatic tumor occurring in a 3-year-old boy. Pediatr Dev Pathol. 2008;11:314-20.

11. Sampelean D, Adam M, Muntean V, Hanescu B, Domsa I. Pancreatic hamartoma and SAPHO syndrome: a case report. J Gastrointestin Liver Dis. 2009;18:483-6.

12. Sueyoshia R, Okazakia T, Lane GJ, Arakawa A, Yao T, Yamataka A. Multicystic adenomatoid pancreatic hamartoma in a child: case report and literature review. Int J Surg Case Rep. 2013;4:98-100.

13. Kim H, Cho CK, Hur YH, Koh YS, Kim JC, Kim HJ, et al. Pancreatic hamartoma diagnosed after surgical resection. J Korean Surg Soc. 2012;83:330-4.

14. Kersting S, Janot MS, Munding J, Suelberg D, Tannapfel A, Chromik AM, et al. Rare solid tumors of the pancreas as differential diagnosis of pancreatic adenocarcinoma. JOP. 2012;13:268-77.

15. Kawakami F, Shimizu M, Yamaguchi H, Hara S, Matsumoto I, Ku Y, et al. Multiple solid pancreatic hamartomas: a case report and review of the literature. World J Gastrointest Oncol. 2012;4:202-6.

16. Addeo P, Tudor G, Oussoultzoglou E, Averous G, Bachellier P. Pancreatic hamartoma. Surg. 2014;156:1284-5.

17. Inoue H, Tameda M, Yamada R, Tano S, Kasturahara M, Hamada Y, et al. Pancreatic hamartoma: a rare cause of obstructive jaundice. Endoscopy. 2014:46:E157-8

18. Yamaguchi H, Aishima S, Oda Y, Mizukami H, Tajiri T, Yamada S, et al. Distinctive histopathologic findings of pancreatic hamartomas suggesting their "hamartomatous" nature: a study of 9 cases. Am J Surg Pathol. 2013;37: 1006-13.

19. Durczynski A, Wiszniewski M, Olejniczak W, Polkowski M, Sporny S, Strzekzyk J. Asymptomatic solid pancreatic hamartoma. Arch Med Sci. 2011;7(6):1082-4.

20. Matsushita D, Kurahara H, Mataki Y, Maemura K, Michiyo H, Satoshi I, et al. Pancreatic hamartoma: a case report and literature review. BMC Gastroenterol. 2016;16:3

21. Kim JH, Park SH, Yu ES, Kim MH, Kim J, Byun JH, et al. Visually isoattenuating pancreatic adenocarcinoma at dynamic-enhanced CT: frequency, clinical and pathologic characteristics, and diagnosis at imaging examinations. Radiology. 2010;257:87-96.

22. Fukukura $Y$, Takumi K, Kamimura K, Shindo T, Kumagae $Y$, Tateyama A, et al. Pancreatic Adenocarcinoma: variability of diffusion-weighted MR imaging findings. Radiology. 2012;263(3):732-40.

23. Luo Y, Dong Z, Chen J, Chan T, Lin Y, Chen M, et al. Pancreatic neuroendocrine tumours: correlation between MSCT features and pathological classification. Eur Radiol. 2014;24:2945-52

24. Kim DW, Kim HJ, Kim KW, Byun JH, Song KB, Kim JH, et al. Neuroendocrine neoplasms of the pancreas at dynamic enhanced $\mathrm{CT}$ : comparison between grade 3 neuroendocrine carcinoma and grade 1/2 neuroendocrine tumour. Eur Radiol. 2015:25:1375-83.

25. Kim JH, Eun HW, Kim YJ, Han JK, Choi Bl. Staging accuracy of MR for pancreatic neuroendocrine tumor and imaging findings according to the tumor grade. Abdom Imaging. 2013;38:1106-14.

26. Yoshioka M, Uchinami H, Watanabe G, Sato T, Shibata S, Kume M, et al. F-18 fluorodeoxyglucose positron emission tomography for differential diagnosis of pancreatic tumors. Spring. 2015:31(4):154

27. Kawada N, Uehara H, Hosoki T, Takami M, Shiroeda H, Arisawa T, et al. Usefulness of dual-phase 18F-FDG PET/CT for diagnosing small pancreatic tumors. Pancreas. 2015:44:655-9. 